\title{
QUANTITATIVE EDX MICROANALYSIS ON VOLATILE ALLOYED NANOPARTICLES
}

\author{
N. Braidy*, S. Dénommée**, C. Kingston**, Z. Jakubek**, B. Simard** and G. A. Botton* \\ *Department of Material Science and Engineering, McMaster University. 1280 Main Street West, \\ Hamilton (ON), L8S 4L7, Canada. \\ **National Research Council of Canada. 100 Sussex Drive, Ottawa (ON), K1A 0R6, Canada.
}

Alloyed nanoparticles (NPs) are attracting significant interest as potential catalysts or as probes for biological or chemical events. Some of the relevant properties of these NPs are critically dependent on their composition. In this regard, energy dispersive X-ray (EDX) spectroscopy in a FEG-TEM has become an essential analytical tool for quantifying the components of individual NPs. However, if one or several components of the nanoparticle are beam-sensitive, the EDX results can be misleading, especially if one element is volatile. In this case, the collection of sufficient X-ray signal to carry adequate quantitative analysis is done at the expense of considerable losses of the volatile component resulting in erroneous analysis. It is possible, however to determine the composition of a NP prior to beam-induced damage based on the analysis of the sublimation rate. This paper presents an example of this simple method for alloyed Au-Ag nanoparticles, a system where Ag is lost during electron irradiation.

Homogeneously alloyed Au-Ag nanoparticles were produced by laser $(532 \mathrm{~nm})$ vaporization of a powder mixture of $\mathrm{Au}$ and $\mathrm{Ag}$ in suspension an aqueous solution of surfactant. Details of this simple method to synthesize alloyed NPs can be found elsewhere [1]. The solution was then centrifuged to remove larger particles and a drop of the diluted solution was left to dry on a holey amorphous carbon film supported on a copper TEM grid. The nanoparticles were analyzed using a JEOL 2010F $(200 \mathrm{kV})$ equipped with a SiLi Ultrathin window energy dispersive detector (Oxford Instrument).

The microanalysis method consisted in recording one EDX spectrum for a constant number of counts (400 counts initially but larger number were used as the particle loss is stabilized) collected under the Au M line (between $2.01 \mathrm{keV}$ and $2.25 \mathrm{keV}$ ). The Au-Ag ratio was then processed for each spectrum using the Cliff-Lorimer Au-Ag k-factors obtained with a microtomed standard (thickness and fluorescence effects were negligible for these NPs) [2]. Since previous measurements demonstrated that the Au count rate is stable, the Ag content could be plotted as a function of a total integrated counts scale that relates to the total electron irradiation time. Using a count scale rather than a time scale makes it possible to account for current drifts and changes in illumination area during analysis. Extrapolating the tendency to the origin yields the initial Ag content of the NP.

FIG. 1a shows a high-resolution TEM micrograph of an Au-Ag NP prior to the acquisition of the EDX spectra series. The NP has a diameter of $\sim 9 \mathrm{~nm}$ and is twinned. For this NP, the first EDX spectrum of the series (FIG. 1b) indicates the presence of $\sim 57 \%$ at. Ag. The values for the $\mathrm{Ag}$ concentration from each of the EDX spectra in the series were then plotted in Figure 2. The Ag concentration is observed to be roughly constant between 50 and $60 \%$ for the 24 first spectra then abruptly decreases to $15-16 \%$. We believe that the initial stage of the curve could be a result of the stabilizing effect of the surfactant. Smaller nanoparticles did not display such behaviour as the Ag content decreased instantaneously upon electron radiation. In these cases, the initial Ag content can 
be determined by extrapolating the curve to the origin. We also noticed that the rate of decrease in $\mathrm{Ag}$ content is higher for smaller NPs because of the larger surface fraction. Also, during irradiation, the NPs shape changes to the most stable configuration.

FIG. 3a illustrates the same NP as in FIG. 1a, following an electron beam irradiation time yielding to 28800 X-Ray counts accumulated for the Au M line. FIG. $3 \mathrm{~b}$ is the final EDX spectrum from the series taken on this NP. It is clear that the NP underwent a considerable volume loss by continuous removal of $\mathrm{Ag}$ due to electron beam irradiation. The volume reduction is in general in good agreement with the amount of $\mathrm{Ag}$ evaporated [3].

\section{[1] J. Zhang et al., Submitted.}

[2] G. Cliff and G. W. Lorimer, J. Microsc. 103 (1975) 203.

[3] The authors wish to acknowledge NSERC and NRC for funding.
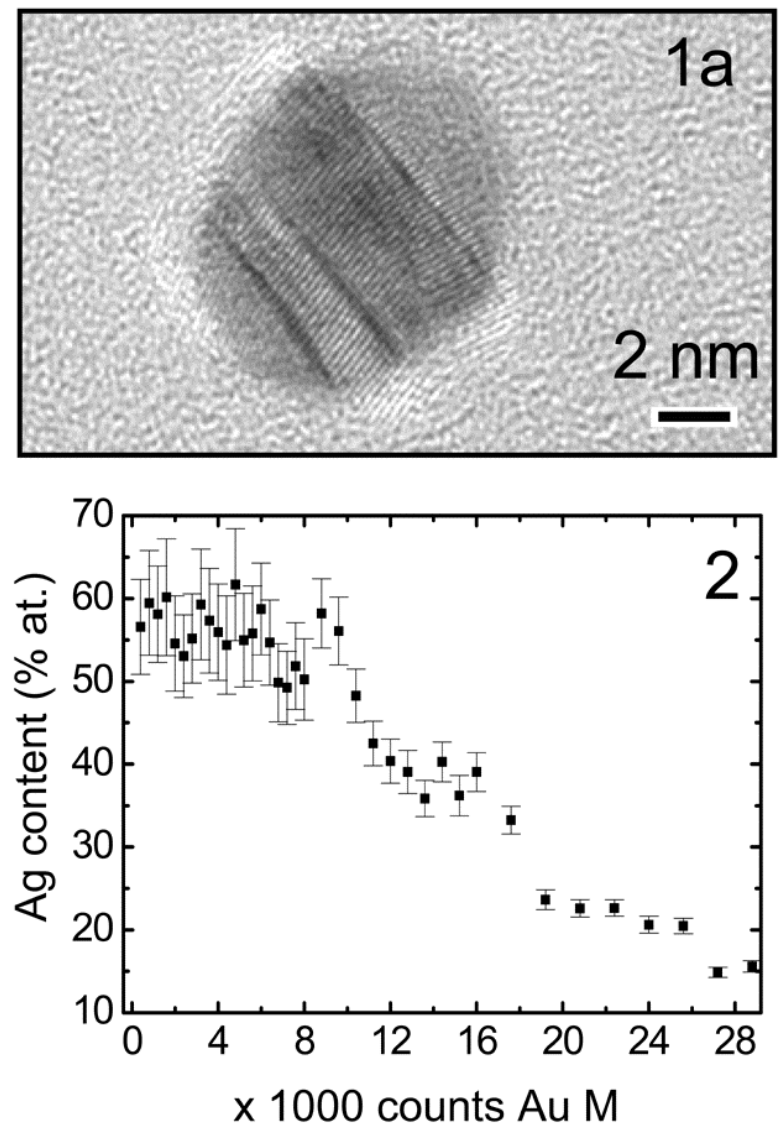

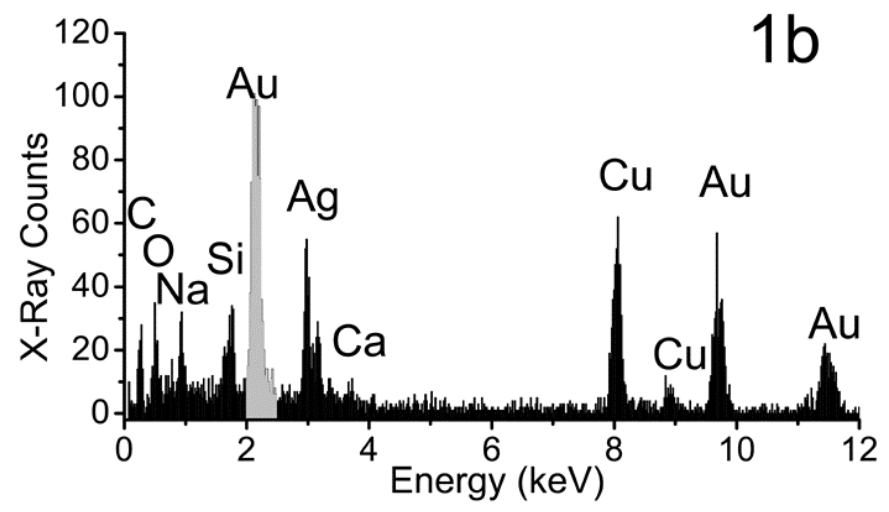

FIG. 1a. HRTEM micrograph of Au-Ag alloyed NP showing twins prior to prolonged electron irradiation.

FIG. 1b. Corresponding EDX spectrum from NP in FIG. 1a. 400 X-ray counts were accumulated within shaded area. Presence of $\mathrm{Na}$ is attributed to the surfactant and $\mathrm{Si}$ is intrinsic to TEM grids.

FIG. 2. Ag content for recorded spectra. Abscissa is the total number of $\mathrm{Au} \mathrm{M}$ counts in the acquisition series and is attributed the exposure of the NP to electron beam.

FIG. 3a. HRTEM micrograph of NP from FIG. 1a after the spectra series of FIG. 2 were recorded. NP size decreased as a result of $\mathrm{Ag}$ evaporation.

FIG. 3b. Corresponding EDX spectrum from NP in FIG. 3a. $1600 \mathrm{X}$-ray counts accumulated within shaded area.

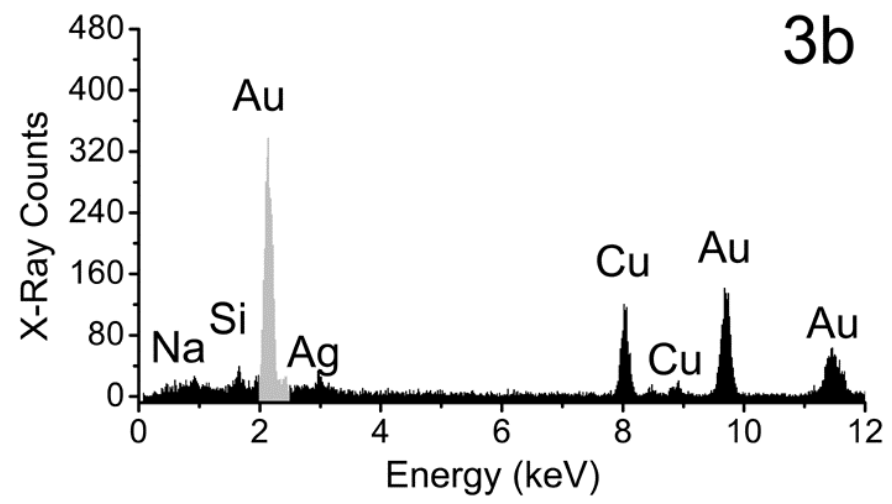

